1. Barresi, A.A. Freeze-drying techniques. New ways to enhance process control and recipe development in pharmaceuticals freeze-drying /A.A. Barresi, D. Fissore, R. Pisano// Pharm Manuf Packing Sourcer. - 2009. - P.36-42.

2. Hottot, A. Experimental study and modeling of freeze-drying in syringe configuration / A. Hottot, J. Andrieu, S. Vessot, et al. // Part I: freezing step. Drying Technol. - 2009. -Vol.27. - P.40-48.

3. Li M.C., Yang G., Zhou X.D., Tseluyko S. S., Perelman Ju. M.//The pathophysiological mechanisms underlying mucus hyper secretion induced by cold temperatures in cigarette smoke-exposed rats. International journal of molecular medicine. 2014. T. 33. № 1. C. 83-90.

4. Severs, N.J. Freeze-facture electron microscopy / N.J. Severs// Nature protocols. - 2007. - Vol.2. - P.547-576

E-mail: agma.agma@yandex.ru gorbunovmed@mail.ru sinowev@mail.ru

\title{
DOI 10.22448/AMJ.2017.3.36-37 \\ COMPARATIVE STUDY ON THE MECHANISM OF BUYANG HUANWU DECOCTION AND ITS MODIFIED RECIPE IN TREATING CEREBRAL ISCHEMIA IN RATS
}

\author{
Haichun Zhou1、Ruizhu Guo2s \\ 1.Second Hospital affiliated to Heilongjiang University of Chinese Medicine 2.Heilongjiang First Aid Center
}

Authors:Postal address:No.411,guogeli Street,Nangang District ,Harbin city, Heilongjiang province

E-mail : zhc801211@163.com $\Delta$ Corresponding author: Postal address:No.109,Satellite Road,Daowai District, Harbin city, HeilongjiangE-mail : 79499743@qq.com

Abstract Objective: to comparatively investigate the effect and mechanism of Buyang Huanwu decoction and modified empirical decoction on the treatment of cerebral ischemia rats

Materials and methods By model establishment from healthy rats, the effect and dosage form of Buyang Huanwu decoction and modified empirical decoction commonly used in the cerebral ischemia clinical treatment were discussed, and DiL was applied to prelabel after effects clarified. After random grouping, MCAO model establishment was conducted after 48h, and the rats were further divided into Buyang Huanwu decoction group (BHD group) and modified empirical decoction group (MED group) according to different therapeutic methods. Research objects were sacrificed at different timings, in order to implement neurological function evaluation, behavioral assessment and pathological inspection. The expressions of FGF-2 and NCAM at infarction border were detected via ISH; cell count of Brdu+/NeuN+ and Brdu+/GFAP+ was performed by immunofluorescent double staining; cell count of $\mathrm{Dil}+/ \mathrm{Brdu}+/ \mathrm{NeuN}+$ and $\mathrm{Dil}+/ \mathrm{Brdu}+/ \mathrm{GFAP}+$ was observed through laser confocalmicroscopy;theexpressionsofNotchlmRNA, Hesl/5mRNA, MashlmRNA and Ngnl/2mRNA were measured by PCR; protein indicators of $p$-Tubulin-III, Notchl and Hesl/5 were quantified via Western blot. By comparison between the collected results, the effects of Buyang Huanwu decoction and modified empirical decoction on the modeling rats (NSC) were observed.

Results and discussion 1.After massive decoction screening, the addition of Asclepiadaceae Heterostemma alatum, Angelica dahurica and grain insects to traditional BHD improved the cerebral infarction area in cerebral ischemia rats as well as the scoring of neurological function $(p<0.05)$;

2.Scores of neurological function evaluation and behavioral assessment were elevated by both treatment methods, however, superior result was obtained in MED group $(p<0.05)$;

3.Infarction border expressions of FGF-2 and NCAM, cell count of Dil+/Brdu+/NeuN+ and Dil+/Brdu+/GFAP+, cell count of Dil+/Brdu+/NeuN+ andDil+/Brdu+/GFAP+,expressionsofNotchlmRNA, Hesl/5mRNA, MashlmRNA and Ngnl/2mRNA, as well as p-Tubulin-III, Notchl and Hesl/5 all indicated therapeutic effects on cerebral ischemia rats by two treatment methods, however, superior result was obtained in MED group $(p<0.05)$.

Superior therapeutic effect of traditional BHD was obtained after

the addition of Asclepiadaceae Heterostemma alatum, Angelica dahurica and grain insects, and the mechanisms might be the involvement of proliferation, migration and differentiation in neural stem cell after cerebral infarction. MED had better therapeutic effect comparing to BHD.

Keywords: neural stem cell; Buyang Huanwu decoction;

References:

[1] Endothelial-to-mesenchymal transition contributes to fibro-proliferative vascular disease and is modulated by fluid shear stress[J] . Moonen Jan-Renier A.J.,Lee Ee Soo,Schmidt Marc,Maleszewska Monika,Koerts Jasper A.,Brouwer Linda A.,van Kooten Theo G.,van Luyn Marja J.A.,Zeebregts Clark J.,Krenning Guido,Harmsen Martin C.. Cardiovascular Research . 2015 (3):15711577.

[2]X.chen.Y.Li.a.E.Kline.etal.Gender and environmental effects on regional brain derived neurotrophic factor expression after experiment- tal traumatic brain injur y[J].Neuroscience.2015.135 (1):11-17.

[3]Fan Guang-ming.Zhang Wen-bin.Zhang Sai.etal.Nerve growth factor comb-

ined with neural stem cell transplantation for treating spinal cord injury in rats[J]. JouRNAl of clinICAI Rehabilitative Tissue Engi36 Амурский медицинский журнал №3 (19) 2017 
[4]Minhua LIN.Lin YaNG.Rong FU.cloning of the Eukaryotic Expression Vector with Nerve Growth Factor in Rats and Its Effects on Proliferation and Differentla tion of Mesencephal Neural Stem cells of Fetal Rats[J].JouRNAl of Huazhong Universt y of Science and Technology(MedICAI Sciences).2008.28(05): 213-216.

DOI 10.22448/AMJ.2017.3.37-37

THE DEMONSTRATIVE STUDY OF MATERIAL BASE AND MECHANISM OF TCM XIAOYAOSAN BASED ON UNDERSTANDING THE ESSENTIAL RELATION OF A TCM PRESCRIPTION AND SPECIAL DISEASE/SYNDROMES

\author{
Haiyang Liu, Ning Zhang, Xia Lei*
}

Hei Long Jiang University of Traditional Chinese Medicine, No.39 Guang Hua Street Qiang Jin District Jia Mu Si 154100 P.R.China

E-mail address: 2577376527@163.com(Haiyang Liu); 327031840@qq.com(Ning Zhang); * Corresponding Author. Tel.: +8618045454111; Fax:0454-8782701. E-mail address: leixia2006@163.com

OBJECTIVE: A new research model "revealing disease nature by the comprehensive application of Metabonomics and Serum Pharmacochemistry of Chinese Medicine, explaining prescription effective mateial basic and mechanism of action on the basis of the relevance between the prescription chemical component and disease nature" is proposed in this thesis. The following results and conclusions are achieved by the study on the Xiaoyao Powder as the research template.

METHODS: 10 batches Xiaoyao Powder HPLC fingerprints were established, 22 common peaks were determined, the silimarity of every batch and common models was above 0.99 .10 batches Xiaoyao Powder volatile components GC-MS fingerprints were establised, 29 common peaks were determined, the silimarity of every batch and common models was above 0.99. The Wistar rat model was established by immobilization stress using bound and fatigue stimulation and solitary ways lasting for 21 days. The model was recognized as model of syndrome of stagnation of liver qi and spleen deficiency from animal characterization, food intake, bodyweight, open field test, novelty suppressed feeding test, sucrose preference test, as well as effect of Xiaoyao powder. The hematuria and biochemical indexes such as D-xylose excretion rate, AST, ALB and CHE was measured and liver injury pathology was observed by HE dye. Furthermore, metabolomics analysis to compare the change trend of metabolic products with normal group.

RESULTS: They showed that established fingerprints were featured by aboundant information, distinct features, high stability and good repeatability, which can effectively monitor the quality of Xiaoyao Powder. 14 blood migration components were identified, No. 3, 9, 10, 14 are the inherent components in blood, the content in blood was increased significantly after taking Xiaoyao Powder. Regarding serum chromatographic fingerprint as direction, relevant drugs as subjects, blood migration components were determined as main component of Xiaoyao Powder effective parts. Viewing from the behavioral observation, comparing with normal group, liver qi stagnation and spleen qi deficiency syndrome group rats and liver injury group rats showed that their expression was tired, their hair scattered, dull yellow and easily fall, they liked gathering together and less activity, listless, their stool was shaped and thin pond. Viewing from liver tissue pathology results, comparing with normal group, hepatic lobule structure disorder occurs among liver qi stagnation and spleen qi deficiency syndrome group rats and liver injury group rats, Local liver cell nucleus atrophy, as well as disintegrating and degenerating, and the infiltration of lymphocyte cells. Viewing from hematuria and biochemical indexes, comparing with normal group, urine D-xylose excretion rate in liver qi stagnation and spleen qi deficiency syndrome group rats and liver injury group rats decreased, AST increased, ALB and CHE decreased.

Viewing from metabolomics analysis, comparing with normal group, Glycine, Butanedioic acid, Propanoic acid, Methionine, Glutamine and Phenylalanine in serum of liver qi stagnation and spleen qi deficiency syndrome group rats and liver injury group rats increased simultaneously, the content of Octadecadienoic acid and Oleic acid decreased simultaneously. Xiaoyao Powder effective parts that formed by serum pharmacochemistry of Chinese Medicine chemical method can improve liver qi stagnation and spleen qi deficiency syndrome and liver injury rats behavioristics, liver tissue pathology and hematuria and biochemical indexes, they can callback the change trend of metabolic products.

CONCLUSION:The common physiology, pathology and metabolic network basis exist in liver qi stagnation and spleen qi deficiency syndrome and liver injury. Xiaoyao Powder effective parts that formed by serum pharmacochemistry of Chinese Medicine chemical method can intervene liver qi stagnation and spleen qi deficiency syndrome and liver injury simultaneously, especially repairing liver cells injury, regulating the content of AST, ALB, and CHE. Metabolomics shows that Xiaoyao Powder plays the role of protecting liver by the regulating of one carbon unit, special metabolism of sulfur contained in amino acids, aromatic amino acids, and three tricarboxylic acid cycle. Metabolomics can build up the nature relationship between prescription internal chemical components and syndrome, disease, serum pharmacochemistry of Chinese Medicine chemical can build up the relationship between prescription internal chemistry components and prescription. Therefore, the combination application of metabolomics and serum pharmacochemistry of Chinese Medicine chemistry can explain the nature relationship of prescription, syndrome and disease, which embodies distinguished advantage in the research of prescription effective mateial basic and mechanism of action.

Keywords: Xiaoyao Powder; Liver qi Stagnation and Spleen qi Deficiency Syndrome; Liver Injury; Metabonomics; Serum Pharmacochemistry of Chinese Medicine 